\title{
Quality in radiotherapy: effective treatment and patient safety
}

\author{
Adelaide Nascimento ${ }^{1}$ and Pierre Falzon \\ Ergonomics Laboratory. Research Center on work Development (CRTD) - Conservatoire National des Arts et \\ Métiers, 41 rue Gay Lussac, 75005 Paris, France
}

\begin{abstract}
The purpose of this article is to understand the way in which medical physicists take into account treatment effectiveness and safety when selecting a treatment plan, with respect to the medical prescription and the technical, human and organizational resources available. Data-gathering was based on the allo-confrontation method: 14 medical physicists from five different treatment centers commented on real treatment plans that had been drawn up by their colleagues. Results show that medical physicists have two means at their disposal to control treatment effectiveness and safety: risk avoidance and risk reduction. Risk avoidance is achieved when conceiving the solution. Risk reduction occurs after the design of the plan and consists in accompanying and assisting the radiographers at the work station where the treatment is carried out.
\end{abstract}

Keywords: safety, effectiveness, quality, radiotherapy

\section{Introduction}

The US Institute of Medicine reports « Crossing the Quality Chasm » (2001) describes the 6 following dimensions of quality in health care: (1) Safety, (2) Effectiveness, (3) Patient centeredness, (4) Timeliness, (5) Efficiency and (6) Equity [1]. This research has considered dimensions 1 and 2 , treatment safety and effectiveness. The goal is to investigate physicists' strategies when a possible conflict between these two dimensions arrives. In this text, « quality » refers to the respect of these two dimensions in radiotherapy treatment.

The objective of radiotherapy is to reduce the volume of the tumor by optimizing the concentration of the prescribed dose within the tumor itself and minimize irradiation of surrounding healthy tissues [2]. In practice, this is carried out through sessions in which fractions of the total dose are administered using various techniques which determine the shape of the radiation beams and the parameters of the machines used in the treatment.

In France, the preparation of treatment plans in radiotherapy requires four types of professionals: radia- tion oncologists, medical physicists, dosimetrists and radiographers. The radiation oncologist is responsible for prescribing the treatment (method and total amount of radiation). Radiographers simulate patient positioning and fashion immobilization devices at the simulation work station. Following this phase, a medical physicist and a dosimetrist work together to design the treatment plan (beam distribution, dose fractionation). This plan is assessed by the radiation oncologist. On the basis of this plan, radiographers define the real conditions for positioning the patient. These last modifications are then checked by a medical physicist. Finally, radiographers administer the actual treatment.

It should be noted that the physicists contribute to the radiotherapy treatment chain at a point situated between the physician's prescription for treatment and the administration of the treatment by the radiographers.

Even though scientific and technological progress has contributed to improving healthcare in terms of clinical effectiveness, the gains for patient health are accompanied by the presence of new risks for patient safety[3-4]. Indeed, medical physicists have access to

\footnotetext{
${ }^{1}$ Corresponding author. Email:adelaide.nascimento@cnam.fr
} 
highly effective techniques, which meet the requirements of the medical prescription, but which present certain risks, especially regarding the difficulties the radiographers encounter in executing the treatment. These situations may lead to conflicts between treatment effectiveness and safety. In some cases a treatment may be very effective from a clinical point of view, but very risky to carry out. If the risks of implementation are reduced, the opposite situation may arise: the treatment will carry fewer risks, but will also be less effective at the clinical level. At some point a decision must be taken to reach the best compromise between effectiveness and safety.

\section{Methods}

Data-gathering was based on the allo-confrontation method [5] : 14 medical physicists from five different treatment centers commented two real treatment plans that had been drawn up by their colleagues. The following were chosen: 1) one case classified as simple, following a protocol: the medical prescription was respected and optimized, and the implementation did not present any particular risks; 2) one case classified as complex, not following a protocol: in order to find an optimal solution for the medical prescription, certain decisions were made. However, this treatment solution, very effective in its virtual version, might create difficulties for the actual implementation of the treatment.

For comparative purposes, we chose subjects in centers of differing legal status (Centre de Lutte Contre le Cancer - CLCC, public and private establishments) and differing resources (cutting edge or conventional). The individual interviews lasted about 30 minutes and were conducted by the first author on the physicists' workstation. The data were collected between March and May, 2009. The interviews were recorded, and then transcribed in full. The transcription of the 14 interviews concerning the two authentic plans provided 28 verbal transcripts (14 subjects $\times 2$ treatment plans). They were subjected to a manual content analysis.

Coding categories have been defined in relation to research needs. Five categories have been set: i) criteria used to evaluate solutions ; ii) assessment of solution acceptability; iii) criteria of acceptability; iv) proposed solutions; v) strategies aiming at reducing risks in implementing the solution. This paper considers the results regarding the first and the last criteria.

\section{Results}

In brief, results show that physicists tend to give prominence to the treatment effectiveness in the virtual phase and to consider safety next. But they also show that physicists use prevention strategies in order to avoid execution errors, in particular by taking into account difficulties radiographers may meet.

When analyzing the treatment plans, medical physicists indicated the criteria they used in order to design or evaluate a plan of treatment. Two criteria were identified: the effectiveness of the treatment and the safety of the treatment.

\subsection{Effectiveness-safety trade-off}

Although the intent to find an optimally effective solution is clearly present amongst medical physicists, the solution is not always easy to implement. The quest for optimization thus becomes the object of a trade-off determined by two main conditions: the resources that the physicists have at their disposal and the technical feasibility of achieving the solution.

The quest for dosimetric effectiveness through innovative techniques is not always carried out without safety risks for the execution of the solution. According to some subjects, the more sophisticated the techniques involved in a solution, the more it will be prone to risks, despite the presence of automatic checks.

You can obtain super sophisticated
doses, with 10 beams of radiation, non
coplanar things, but the more
sophisticated the things you do, the
greater the risk of error. P7, Center A

In these situations, the ideal solution for a safe and effective treatment cannot be implemented:

- Either one reduces the risks in executing the solution, but then there is loss in effectiveness. This type of compromise is chosen by a few subjects (3/14) who, although aware of the loss in terms of treatment effectiveness, give priority to a gain in safety:

For my part, I prefer a very simple, old-fashioned treatment - something that was already being done 20 years ago, with a square field, and which is perhaps not optimal, but which I know has stood the test of time - to something new and very sophisticated, but which is often not without its risks.. P2, Center A. 
- $\quad \ldots$ or one maintains the effectiveness of the treatment, despite the increased risk of misimplementation when the solution is carried out.

This last type of compromise is favored by the majority of the subjects (11/14). It involves accepting certain risks, which need to be taken into account when implementing the solution, in exchange for the certitude of the clinical effectiveness of the treatment.

If we can do something that is optimal
and it is possible to implement it, even
if it is going to be difficult, we do it. At
least in the cases I've seen up to now,
that's what we have always done. For
the moment, it is more the effectiveness
of the treatment that is important, even
if it is longer and more complicated at
the work station. We prefer to spend
more time on it, as long as it is for the
good of the patient. P11, Center B.

Nevertheless, the analysis of the data indicated that strategies were employed by physicists to enhance effectiveness and safety of treatments. Actually, physicists have some means of control at their disposal in order to deal with the risks. These involve anticipating the difficulties of execution which the radiographers may encounter and setting up strategies to prevent risks, thus allowing them to control the optimal effectiveness and safety of the treatments. The next section presents these risk prevention strategies.

\subsection{Prevention strategies designed to guarantee quality care in radiotherapy}

When medical physicists consider the solution to be risk-prone, they anticipate the risks. The subjects were unanimous in mentioning strategies aimed at facilitating the work carried out by the radiographers at the end of the treatment chain, and thus to reduce the risk of mis-implementation.

On paper, you can be under an illusion
of precision. Obviously, on paper the
cuts don't move, the patient doesn't
breathe, all is well. But in reality, this
is not the case, and that has to be taken
into account. P7, Center A

To do this, they use of two main types of prevention strategies that allow them to act upon the criteria for the safety of the treatment in order to approach the desired optimal effectiveness: avoiding the risks and assisting the radiographers (see Table 1).

\subsubsection{Avoiding the risks}

Once the virtual design for an optimal solution has been produced, the criteria that the subjects most often refer to are those that take into account the sources of risk in implementing the solution. The aim of verifying these criteria is to ensure that a virtual design for an optimal solution will still be optimal in a real situation. Indeed, physicists reduce the factors that could lead to errors at the source - i.e. the virtual planning stage - either by avoiding the use of "high-risk" work stations (ones that have an overload of patients, old machines or new radiographer) for complicated treatments, or by limiting the complexity of certain parameters of radiation. In addition, physicists can try to improve the comfort of the patient in order to keep them from moving in a way that would compromise the correct delivery of the dose to the prescribed volume. Thus, the physicists check the conformity of at least three sources of risk: the technical factors, the human and organizational factors, and the patient.

Half of the physicists mentioned that they adjust certain radiation parameters to avoid "confusing" the radiographer or to facilitate their work. The means used are designed especially to reduce the time involved in manipulation and to minimize errors commitment.

\subsubsection{Assisting the radiographers}

The strategies for assisting the radiographers include not only oral and/or written transmission of solution designs, but also the presence of physicists or dosimetrists by the operator's side when the solution is first implemented. These strategies aim to control the sources of risk that were not already taken into account during the virtual conception.

\subsubsection{The oral and/or written transmission of solution designs}

In all, 13 of the 14 physicists mentioned that when a solution is complicated or unusual, the routine instructions for implementing the treatment that are provided in the file are re-enforced, and any possible changes are indicated. This is a way of anticipating certain difficulties that the radiographers could encounter during a treatment session. 


\subsubsection{Presence during the first implementation of a complicated solution}

For the non-standard cases, giving instructions alone is not sufficient to reduce the risks involved in carrying out a complicated solution. As a matter of fact, nine of the 14 subjects mentioned that one of the ways to reduce the complexity of a solution was to be present for the first implementation, that is, to be present in the $\mathrm{x}$-ray room with the radiographers, in order to better control the risks.

Table 1

Risk prevention strategies [6]

\begin{tabular}{|c|c|c|c|c|}
\hline \multirow{12}{*}{ 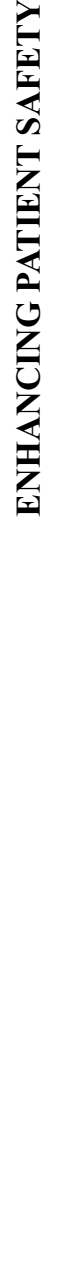 } & \multirow{8}{*}{ Avoiding risks } & \multirow{4}{*}{$\begin{array}{l}\text { Accounting for } \\
\text { technical factors } \\
\qquad(\mathrm{n}=8)\end{array}$} & \multirow{2}{*}{ Type of machine } & $\begin{array}{l}\text { Avoid sending complicated } \\
\text { treatments to old work stations }\end{array}$ \\
\hline & & & & $\begin{array}{l}\text { Limit the complexity of manually } \\
\text { controlled radiation parameters }\end{array}$ \\
\hline & & & Radiation parameters & $\begin{array}{c}\text { Adjust certain risk-prone } \\
\text { parameters despite presence of } \\
\text { automatic controls }\end{array}$ \\
\hline & & & Quality and type of contention & $\begin{array}{l}\text { Take into account to determine } \\
\text { length of session }\end{array}$ \\
\hline & & \multirow{3}{*}{$\begin{array}{l}\text { Taking human and } \\
\text { organizational } \\
\text { factors into account } \\
\qquad(\mathrm{n}=6)\end{array}$} & Radiographers' experience & $\begin{array}{l}\text { Avoid sending a complicated } \\
\text { treatment to a work station where } \\
\text { the radiographers are novices }\end{array}$ \\
\hline & & & Workload at the station & $\begin{array}{l}\text { Avoid sending a complicated } \\
\text { treatment to a work station where } \\
\text { there is an overload of patients }\end{array}$ \\
\hline & & & $\begin{array}{l}\text { Quantity of complicated cases } \\
\text { per day }\end{array}$ & $\begin{array}{l}\text { Avoid sending a complicated } \\
\text { treatment to a work station where } \\
\text { there is a large number of } \\
\text { complicated treatments per day }\end{array}$ \\
\hline & & $\begin{array}{l}\text { Taking patient } \\
\text { comfort into } \\
\text { account } \\
(n=6)\end{array}$ & $\begin{array}{l}\text { Position difficult to } \\
\text { maintain/Patient suffering } \\
\text { from pain/Children }\end{array}$ & $\begin{array}{l}\text { Avoid positions that are difficult to } \\
\text { maintain for a long period, } \\
\text { especially for patients suffering } \\
\text { from pain or for children }\end{array}$ \\
\hline & \multirow{4}{*}{$\begin{array}{c}\text { Assisting } \\
\text { Radiographers }\end{array}$} & \multirow{3}{*}{$\begin{array}{c}\text { Oral or written } \\
\text { transmission } \\
(\mathrm{n}=13)\end{array}$} & \multicolumn{2}{|c|}{ Warning messages indicating a non standard treatment } \\
\hline & & & \multirow{2}{*}{\multicolumn{2}{|c|}{$\begin{array}{c}\text { Instructions indicating a call for help } \\
\text { Instructions for positioning }\end{array}$}} \\
\hline & & & & \\
\hline & & $\begin{array}{l}\text { Presence of the } \\
\text { medical physics } \\
\text { team } \\
(\mathrm{n}=9)\end{array}$ & \multicolumn{2}{|c|}{$\begin{array}{c}\text { Oral instructions, verification of the reproducibility of the simulated } \\
\text { positioning }\end{array}$} \\
\hline
\end{tabular}

\section{Conclusions}

In cases of conflict between the effectiveness and the safety of a solution, the majority of the physicists give priority to effectiveness, achieved by means of a treatment plan. In designing this solution, the ease of execution for the radiographers is not a factor that is taken into consideration. Safety is handled in a second step, by facilitating or assisting the implementation of the treatment. In particular, the results show the important role of oral and written transmissions, and of the presence of a physicist/dosimetrist at the radiation workstation when complicated treatments are administered. These strategies are shared by the 
majority of physicists and are a sign of a strong culture of effectiveness, based on the optimization of medical protocols and associated with a culture of safety, founded on the prevention of risks at the workstation. The former is prescribed by the formal rules of radiation, while the latter is not formalized and belongs to the realm of practical knowledge about the radiographers' situations, organization and work.

Adverse outcomes are too frequently attributed to those who provide radiotherapy. Contrary to this, the findings of this study indicate consistent concern for and efforts to enhance patient safety while providing effective treatment by those who plan and implement radiotherapy. Thus, when an adverse outcome occurs, rather than automatically blaming the technician, others possible contributing factors, such as the design of the radiotherapy device, are candidates for consideration.

\section{References}

[1] D. M. Berwick, "A User's Manual For The IOM's 'Quality Chasm' Report. Patients' experiences should be the fundamental source of the definition of "quality.," Health affairs, vol. 21, 2002.

[2] J. P. Le Bourgeois, "Place de la radiothérapie dans la stratégie thérapeutique des cancers.," in Techniques d'irradiation des cancers : la radiothérapie conformationnelle., J. J. Mazeron, et al., Eds., ed Paris: Ed. Maloine, 2006, pp. 13-19.

[3] ANAES, "Principes méthodologiques pour la gestion des risques en établissement de santé," Rapport de l'Agence Nationale d'Accréditation et d'Evaluation2003.

[4] P. Carayon, et al., "Work system design for patient safety: the SEIPS model," Qual Saf Health Care, vol. 15 , pp. 50-58, 2006.

[5] V. Mollo and P. Falzon, "Auto- and allo-confrontation as tools for reflective activities," Applied Ergonomics, vol. 35, pp. 531-540, 2004.

[6] A. Nascimento, "Produire la santé, produire la sécurité. Développer une culture collective de sécurité en radiothérapie.," Thèse de Doctorat en Ergonomie Thèse de Doctorat en Ergonomie, Laboratoire d'Ergonomie, Conservatoire National des Arts et Métiers, Paris, 2009. 